\title{
WIRELESS BODY AREA NETWORK (WBAN) FOR HEALTH MONITORING SYSTEM USING GENETIC ALGORITHM AND FUZZY LOGIC
}

\author{
Meetali Thakur \\ Student M.Tech, ECE \\ RIEIT, Railmajra \\ Punjab,India
}

\author{
Er.Parvinder Singh \\ Associate Professor, ECE, \\ RIEIT, Railmajra \\ Punjab,India
}

Abstract: WBAN (Wireless Body Area Network) is one of the mostly used real time networks can be explained by certain limitations and constraints. The difficulties of the network occur because of the environmental constraints, device restrictions and architectural constraints. These problems also lift up various issues associated to network life, reliability and security. It is an automatic system utilized for the supervision of patients in circle condition. In the research, we have designed a network area with length and width of size $1000 \times 1000$ and 50 numbers of sensor nodes and the procedure is being iterated for almost six times. The research is being simulated in MATLAB environment. Parameters, namely, energy Consumption and Throughput have been used for checking the performance of the research. Optimization algorithm that is Genetic Algorithm is utilized for improving the performance of the parameters. The extraneous data is being removed by using Fuzzy logic classifier.

Keywords: wireless body area network (WBAN); genetic algorithm; Fuzzy logic; MATLAB

\section{INTRODUCTION}

Patient data monitoring is considered as a leading issue for disease and health management. With this system, health information is significant when some patient is in serious condition or has some heart fetal case. Advancement for developing the sensors on patient body has been made in which the sensors are attached with the patient body without the requirement of gels [1].
The patient can be monitored by two types, namely, wired and wireless. For the wired system, the cables are linked with sensors and then the collected data is executed and later stored in the $\mathrm{dB}$ (database). For the wireless monitoring system, not a single wire is integrated with the data and is transferred with the remote station by means of radio waves [2].

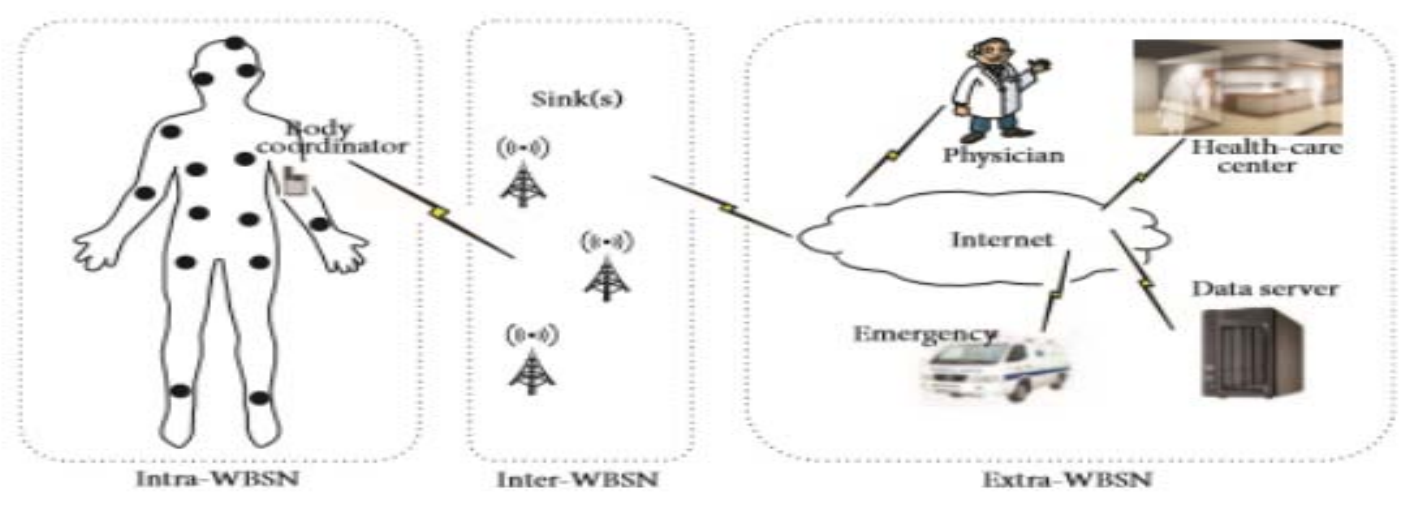

Figure 1. Architecture of WBAN

In the above figure, the sensors are being located on human body. The sensors collect the data and send to the sink which is a base station that could be an android phone/laptop. Now, by means of the radio link, the integrated information is transferred to the remote station that might be a healthcare centre, physician, and emergency ambulance and is stored in database for future use [3].The main issue arises while execution is the battery power and sensor size that should be resolved. It become a challenge to send a heterogeneous data and utilizes multipurpose applications for reaching the mass market. The data can be integrated from one sensor to other sensor via Bluetooth, Radio frequency signals, ultra wide band, Zigbee and many more [4]. The initial transmission of data can be possible by means of Bluetooth and consumes more power from the sensors. The main problems being analyzed in the trending approach is raised when the authors dint pay more attention to the data storage because the devices are less capable for storing the data. Therefore, a requirement is there for developing a system that would be considered as a solution for the mentioned problem.

The evaluated solution would be dependent on QoS (Quality of Service) Parameters. The expected parameters would be:
i. Throughput
ii. End to End delay 


\section{iii. Energy Consumption \\ iv. Bit Error Rate}

\section{MATERIALS AND METHODS}

This research has focused on GA (Genetic algorithm) for the optimization of the results. The classification is done by using Fuzzy logic and is explained below:

\section{A. Genetic Algorithm}

GA is a technique utilized for solving the issue of optimization by biological evolution. At every step, it chooses the population randomly an individual nearby the parents and later generates the children for the future generation. The mentioned procedure is iterated till an enhanced solution is obtained. GA mainly includes three main rules, namely, Selection rule, Crossover rule and Mutation Rule.

- $\quad$ Selection Rule

It chooses the individuals, termed as parents responsible for to the population for the subsequent generation.

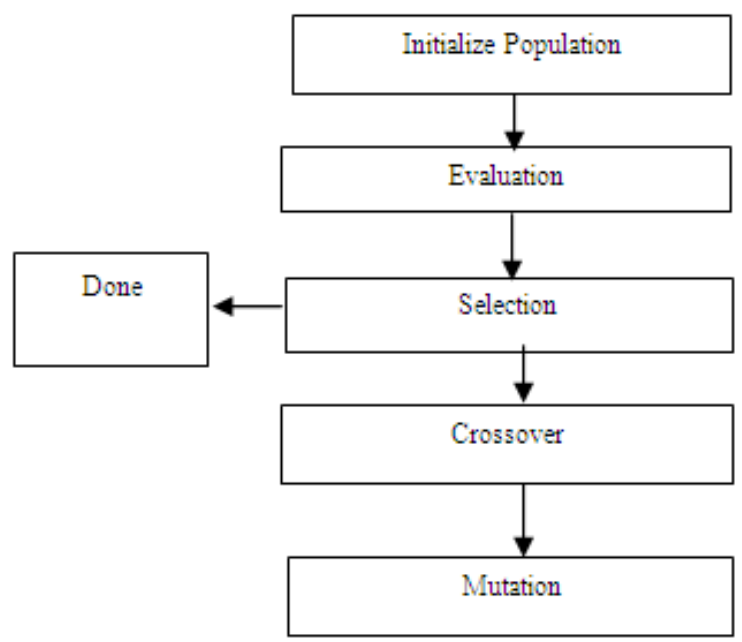

Figure 2. Flowchart of Genetic algorithm

\section{- Crossover rule}

It combines two parents to produce two children for the subsequent generation.

- Mutation rule

It is utilized to execute random variations to individual parents to produce children [5].

\section{B. Fuzzy logic}

It is a method that is based on degree of truth rather than on $\mathrm{T}$ (True) or $\mathrm{F}$ (False) on which the current computer are dependent. The implementation of fuzzy logic is as same as human brain working. It is being introduced by Lotfi Zadeh in 1965.

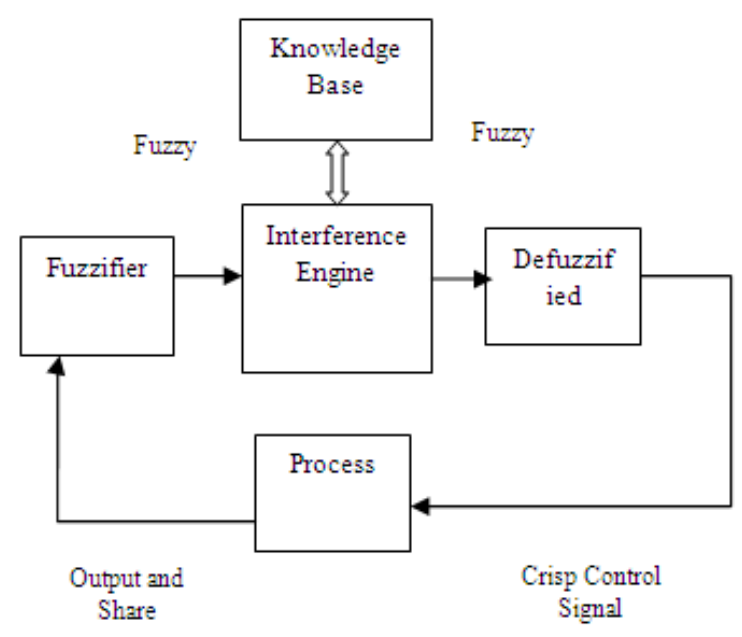

Figure 3. Architecture of Fuzzy controller

\section{RELATED WORK}

M. Li et al. [6, 2010] focused on privacy and data security in WBAN (Wireless body area network). The data security for data transmission and its storage in $\mathrm{dB}$ is being studied. $\mathrm{M}$. Roy [7, 2017] designed WBAN on the basis of two hop cost energy effective routing protocol. The proposed system resolves the sensor's energy drainage and later has routing decisions. The research has been simulated in Castalia simulator. Z. Ling et al. [8, 2017] reviewed energy harvesting for point to point communication for Wireless Body Area Network. An author takes two conditions for data transmission, namely, normal and abnormal situations. For normal circumstances time switching protocol is used whereas for abnormal circumstances enhanced power splitting and radios or time switching are used. H. MosavatJahromi et al. [9, 2017] investigated a spectral effective of a communication link in Wireless Body Area Network utilized for storing energy. Authors has used policies of power management and used two scenarios, namely, single and dual hope. Y. Kim et al. [10, 2016] presented an algorithm being utilized for solving the inter-reference problem occurred in Zigbee based WBAN. M. M. Alam, et al. [11, 2016] presented a heuristic dependent method for reconfiguring the sensor nodes wake up schedule in WBAN. The protocol utilized is dependent on the adaptive algorithm. N. Boujnah and F. Mars [12, 2016] presented a technique for reducing the energy consumption for increasing the sensor nodes battery life. D.Kavitha et al [13, 2016] enhanced the battery lifetime and throughput of sensor nodes by utilizing WBAN. The research has been carried out in NS-2 Simulator. Pervez Khan et al [14, 2010] focused on diagnostic model for determining metrics performance like mean frame service time, energy consumption, and normalized throughput. The authors have developed a prototype termed as Markov Chain model for different accessing phase depends upon the user's priority. D. B. Jourdan and O. L. de Weck [15, 2004] presented a MOGA (multi objective genetic algorithm) for performance parameters optimization. 


\section{SIMULATION MODEL}

The research is based on wireless area network (WBAN) used for the monitoring of patients at remote place by utilizing GA and Fuzzy logic. The works is being tested on parameters, namely, throughput, BER (Bit Error rate), Energy consumption and end to end delay and are explained below:

\section{A. Throughput}

It is termed as the total number of packets sends in the simulation time.

It can be explained mathematically as:

$$
\text { Throughput }=\frac{\sum \text { Packtssent }}{\text { Totaldatapackets }}
$$

\section{B. BER (Bit Error rate)}

BER is defined as the number of bits transmitted per unit time.

$$
\begin{aligned}
& \mathrm{BER}=\frac{\text { Bit errors }}{\text { total number of transferred bit during an interval }} \\
& \text { BER can be described in percentage. }
\end{aligned}
$$

\section{Energy Consumption}

Energy consumption is termed as the total energy being transferred by every node in WBAN at varied network layers.

It is defined mathematically as below:

Energy Consumption

$$
=\sum_{\mathrm{i}=0}^{\mathrm{n}-1}(\text { Energy_consumed_by_node }(\mathrm{i}))
$$

\section{End to end delay}

In the mathematical term, end to end delay is defined as the total time being elapsed for the packet data transmission.

Dend-end $=$ Dtrans + Dprop + Dproc

Where Dend-end= End-To-End Delay

Dtrans $=$ Transmission Delay
Dprop= Propagation Delay

Dproc $=$ Processing Delay

This research work has a problem for the storage of data because the devices have less capacity for storing the data. Second problem is the sensor node battery life. More transmission of data requires more energy and therefore, sensor node's life degraded. Therefore, there is a requirement for developing a system that could be a solution for the mentioned problem. The evaluation solution is dependent on QOS (Quality of Service) Parameters as discussed above.

The following are the steps that demonstrate the different phases that need to be accomplished.

Step 1: Design WBAN by utilizing network area of height and width of $1000 \times 1000$.

Step 2: Initialize the sensor nodes in the WBAN. In the proposed work, we are implementing 50 number of sensor nodes.

Step 3: The WBAN coverage area is initialized by utilizing source node and destination node in the WBAN.

Step 4: With the routing protocol, the route is being discovered by the data transmission of source node towards destination node.

Step 5: For optimizing the route, the best route optimization algorithm named as Genetic algorithm is applied.

Step 6: If the GA fitness function is true as per the fuzzy logic rule set then a novel route is developed between source and destination otherwise the node is rejected and checks another node in the WBAN.

Step 7: A best possible is created among source and destination, the performance metrics like energy consumption, bit error rate, throughput, and delay in the network has been measured.

\section{SIMULATION RESULTS}

After the simulation, below results has been obtained: 


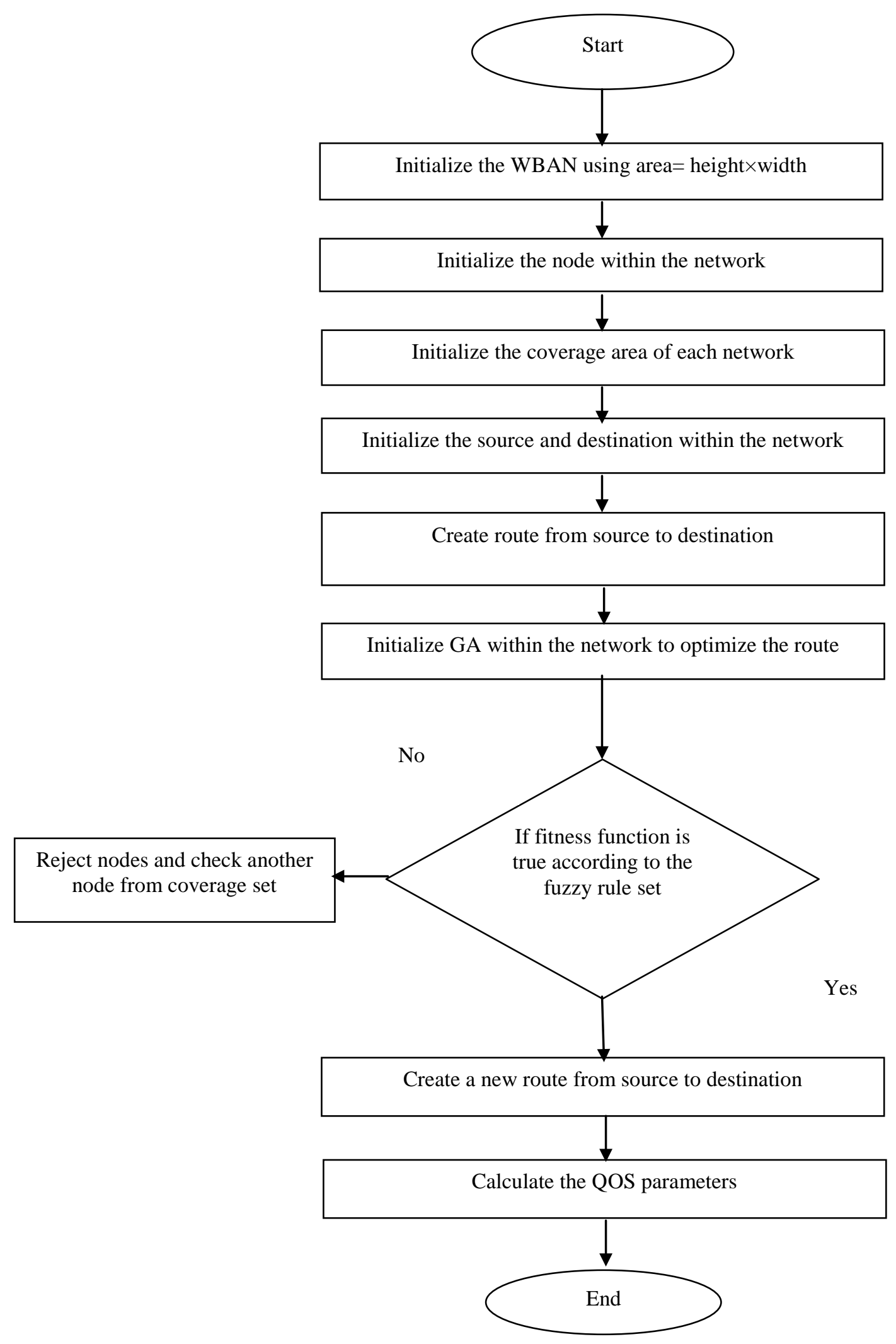

Figure 4. Proposed Methodology Flowchart 

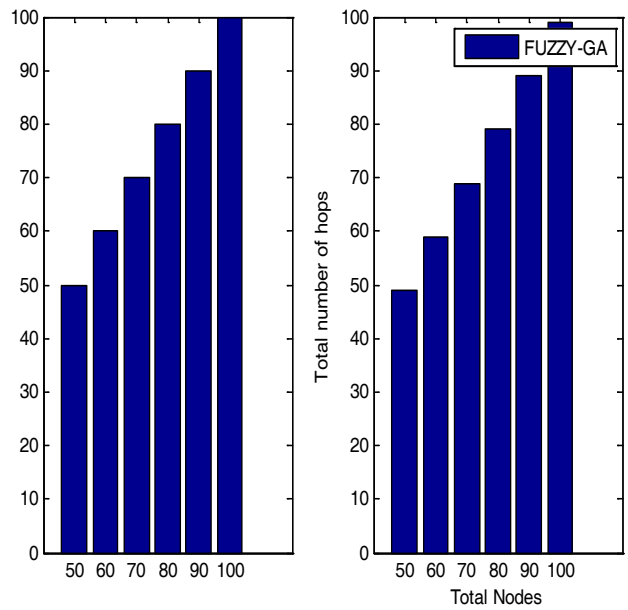

Figure 5. Number of hops for total number of nodes

As shown in the above figure, two images have been displayed. The first image is depicting the algorithm with no optimization and the hop number for each iteration is more that is equal to 50 numbers of hops. The second image is showing a case when GA and Fuzzy has been applied and the hops get reduced. In this case, the hops become equal to 50 almost. It is being concluded from the above figure that in the case of without optimization, energy optimization is more and is less when optimization algorithms has been applied.

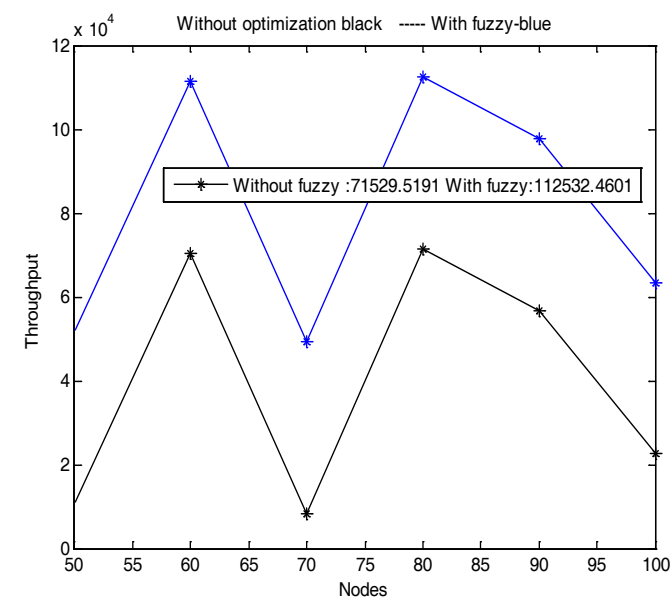

Figure 6. Throughput w.r.t number of nodes

In the above figure, iterations numeral along $\mathrm{x}$-axis and throughput has been shown along y-axis. The work being proposed is simulated by six iterations for determining the best value. Here, black line defines the value obtained when no optimization algorithms is utilized and blue line defines the values occurred for with optimization. The maximum value obtained in case of throughput without fuzzy is 92433.6095 and for with fuzzy is 130931.5552. Also, the average value for 100 number of nodes for with optimization and without optimization are $4.86 \times 104$ and $9.7 \times 104$ respectively.

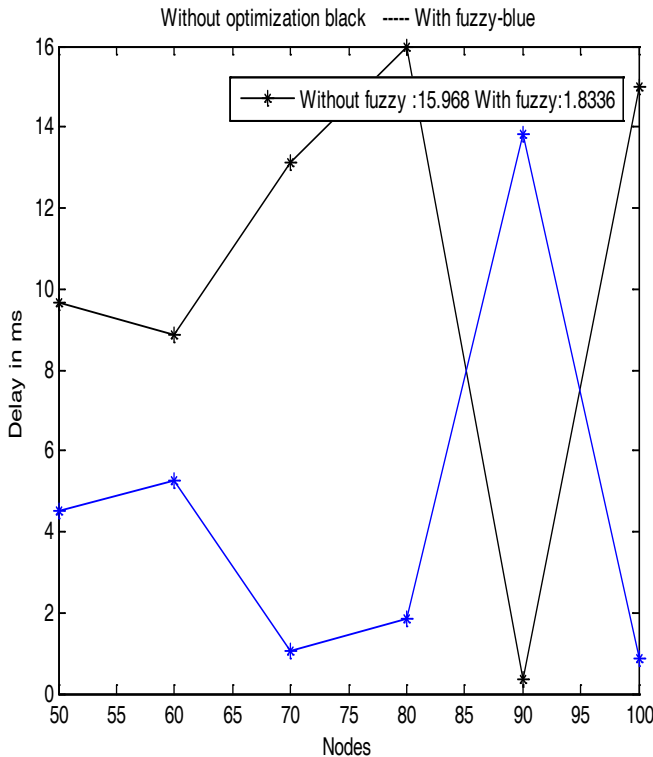

Figure 7. Delay with and without fuzzy

For delay, the average value executed for with and without optimization is 5.7 and 12.7 respectively. Therefore, it is being concluded that when classification and optimization algorithms are executed to the network, delay get reduced whereas in case of without optimization, delay network for 100 nodes is 12.7 .

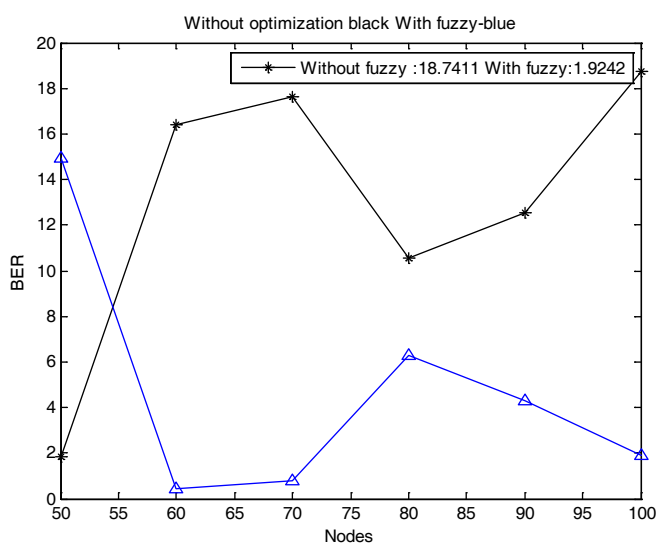

Figure 8. BER with and without optimization

Above figure defines that bit error rate is reduced when optimization algorithm is applied. The BER with optimization and without optimization are 6.1 and 16 respectively.

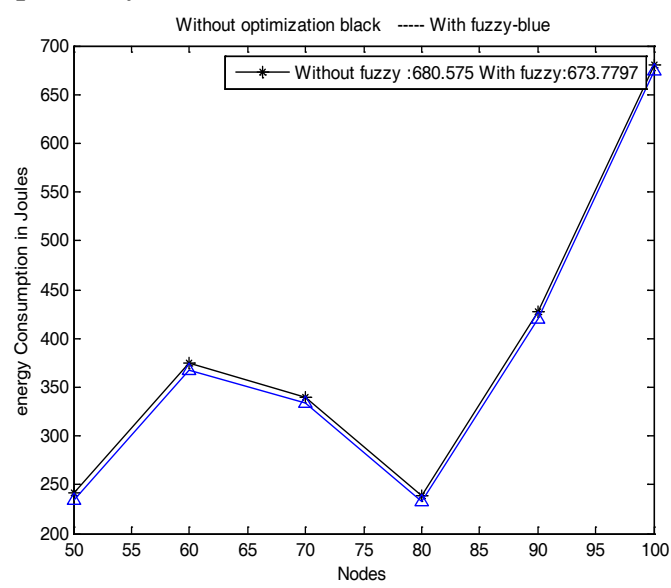

Figure 9. Energy consumption with and without optimization 
Energy consumption is less when optimization algorithm is applied in the research. The consumption for energy in case of optimization is 482 and is 480 when optimization is not applied.

\section{CONCLUSION}

WBAN has been proposed in the research that is utilized for supporting on demand, normal traffic and emergency with the help of communication links. In the work, the radio links are utilized for transmitting the integrated information from one node to other node by utilizing appropriate routing protocol. The routing protocols determine the suitable and smallest route by which the data transmission can be less costly, consumption of energy is less and less error rate at the destination. The simulation is being carried out in MATLAB simulation tool and parameters, namely, throughput, end to end delay, energy consumption and bit error rate has been calculated. With the utilization of optimization and classification algorithms, the performance metrics has performed well.

Future lies in using the hybridization of optimization techniques for enhancing the WBAN system performance by number of classifiers. GA (Genetic Algorithm) can be integrated with other optimization algorithms, namely, PSO (Particle Swarm optimization, ACO (Ant Colony Optimization), BFO (Bacterial Foraging Optimization) and so on for providing the better and enhanced results.

\section{REFERENCES}

[1]. Chen, Min, et al, "Body area networks: A survey,” Mobile networks and applications, vol.16, pp. 171-193, 2011.

[2]. J. Anand and D. Sethi, "Comparative analysis of energy efficient routing in WBAN," 3rd International Conference on Computational Intelligence \& Communication Technology (CICT), Ghaziabad, India, pp. 1-6, 2017.

[3]. A. A. Pramudita, "Input impedance model of planar dipole antenna for Wireless Body Area Network (WBAN)," 22nd Asia-Pacific Conference on Communications (APCC), Yogyakarta, pp. 66-69, 2016.

[4]. A. R. Syed and K. L. A. Yau, "On Cognitive Radio-based Wireless Body Area Networks for medical applications,” IEEE Symposium on Computational
Intelligence in Healthcare and e-health (CICARE), Singapore, pp. 51-57, 2013.

[5]. R. Chávez-Santiago, A. Khaleghi, I. Balasingham and T. A. Ramstad, "Architecture of an ultra wideband wireless body area network for medical applications,"2nd International Symposium on Applied Sciences in Biomedical and Communication Technologies, Bratislava, pp. 1-6, 2009.

[6]. M. Li, W. Lou and K. Ren, "Data security and privacy in wireless body area networks," in IEEE Wireless Communications, vol. 17, no. 1, pp. 51-58, February 2010.

[7]. M. Roy, C. Chowdhury and N. Aslam, "Designing an energy efficient WBAN routing protocol," 9th International Conference on Communication Systems and Networks (COMSNETS), Bangalore, 2017, pp. 298-305, 2017.

[8]. Z. Ling, F. Hu, L. Wang, J. Yu and X. Liu, "Point-to-Point Wireless Information and Power Transfer in WBAN with Energy Harvesting,” in IEEE Access, vol. 5, no. , pp. 86208628, 2017.

[9]. H. Mosavat-Jahromi, B. Maham and T. A. Tsiftsis, "Maximizing Spectral Efficiency for Energy HarvestingAware WBAN," in IEEE Journal of Biomedical and Health Informatics, vol. 21, no. 3, pp. 732-742, May 2017.

[10].Y. Kim, S. Lee and S. Lee, "Coexistence of Zigbee-Based WBAN and Wi-Fi for Health Telemonitoring Systems," in IEEE Journal of Biomedical and Health Informatics, vol. 20, no. 1, pp. 222-230, Jan. 2016.

[11].M. M. Alam, E. Ben Hamida, O. Berder, D. Menard and O. Sentieys, "A Heuristic Self-Adaptive Medium Access Control for Resource-Constrained WBAN Systems," in IEEE Access, vol. 4, no. , pp. 1287-1300, 2016.

[12].N. Boujnah and F. Mars, "Energy saving in WBAN networks under rate constraints," 4th International Conference on Control Engineering \& Information Technology (CEIT), Hammamet, pp. 1-4, 2016.

[13].D.Kavitha, Dr.T.V.P.Sundararajan, P.Hamsagayathri, M.Gunavathi, "Energy Efficient transmission path for wireless body areas network”, IRJET, Vol 3, Issue 4, Pp 1-4, 2016.

[14].Khan, Pervez, Niamat Ullah, Md Nasre Alam, and Kyung Sup Kwak., "Performance Analysis of WBAN MAC Protocol under Different Access Periods" International Journal of Distributed Sensor Networks, vol. 11. 2010.

[15].D. B. Jourdan and O. L. de Weck, "Layout optimization for a wireless sensor network using a multi-objective genetic algorithm,” IEEE 59th Vehicular Technology Conference. VTC 2004-Spring (IEEE Cat. No.04CH37514), vol.5. pp. 2466-2470, 2004. 